\title{
Effect of sequential blocking of similar trigrams on free and serial recall'
}

\author{
WILLIAM F. BATTIG \\ UNIVERSITY OF MARYLAND
}

Recall performance on 15-item lists consisting of three letter orders of each of five three-letter anagrams was markedly facilitated if the letter orders of each set were blocked rather than unsystematically ordered during presentation, and'or Ss were permitted free recall of the items in any order. The blocking effect was larger in magnitude, and also eliminated the superiority of free over serial recall.

Studies of clustering and organizational processes in free recall of verbal materials have focussed primarily on semantic or assoutative bases for grouping or categorization (Tulving, in press), whereas little attention has been directed toward the investigation of organization on the basis of formal similarity. In the present study, maximal similarity was produced by including in the same list sets of three different letter orders of the same three letters. The purpose was to determine (a) whether it was possible for Ss to learn such lists, and (b) the extent to which such learning could be facilitated through the sequential blocking of similar items in adjacent serial positions. Through the inclusion of conditions involving both free and serial recall, the design also permitted the assessment of the generality of Waugh's (1961) finding that serial recall of items in order becomes equivalent or superior to free recall on later trials.

\section{Method}

All Ss learned the same 15-item list consisting of three letter orders of each of five three-letter anagrams, each of which could be rearranged to form a common English word. The five sets of items were (ISX, XSI, IXS); (VNA, NVA, AVN); (IPZ, IZP, PZI); (DMU, UDM, MDU); and (VYI, IYV, YVI), all selected so as to be maximally alike in rated and actual learning difficulty (Battig, Merikle, \& Schild, 1965). Under blocked (B) conditions, the 15 items were presented on each trial in the same order as given above. Under unblocked (U) conditions, the item order on each trial was:

\section{ISX-NVA-PZI-UDM-YVI-AVN-XSI-DMU- VYI-IZP-MDU-IXS-VNA-IPZ-IYV,}

which provided that each third of the list contained one item from each set and that no two items from a given set were separated by fewer than two other items.

All Ss were run in groups in a large classroom, with items being projected on a large screen at the front of the room. Each of 10 successive trials began with the 3-sec. presentation of each of the 15 items in the same B or $U$ order, followed by $1 \mathrm{~min}$. for attempted written recall on a page containing 15 numbered lines in a prepared response booklet. Both the B and $U$ groups were approximately evenly divided into free-recall (FR) and serial-recall (SR) conditions, depending upon whether the instructions on the first page of the booklet (and repeated at the top of each subsequent page) specified that $S$ was to recall the items "in any order that they occur to you" or "in the same order in which they were given." To provide for equal numbers of Ss (18) in each of the four groups (B-FR, B-SR, U-FR, and U-SR), it was necessary to discard randomly four Ss from the U-FR group.

Following the tenth trial, the lastpage of the response booklet contained in an unsystematic order all six possible letter orders for each of the five three-letter sets, on which Ss were instructed to check those letter orders which they recognized as having appeared in the previous list.

Results

Significantly $(p<.001)$ more total correct items were recalled under $B(118.6)$ than $U(88.5)$ conditions, and also under FR (109.8) than SR (97.3) conditions. Both main effects are attributable primarily to the marked inferiority of the U-SR group (74.0) to B-SR (120.7), B-FR (116.6) and U-FR (102.9) groups, which led also to a significant $(p<.001)$ B-U by FR-SR interaction. However, the inferiority of U-FR to both B-FR and B-SR conditions was also significant $(p<.05)$. Examination of the trial-by-trial performance curves revealed these group differences to be consistent over trials, except that U-FR performance on the first trial (6.7), and to a lesser extent also B-FR (6.0), were both slightly but insignificantly superior to B-SR (5.7). Analyses of the improvement from the first five to the last five trials showed this to be significantly $(p<.05)$ greater for $B$ than $U$ conditions and for SR than FR conditions. Although the interaction did not approach significance, these differences reflected primarily the lesser improvement shown by the U-FR group, whereas the superiority of B-SR over both B-FR and U-SR groups did not approach significance.

Since $31.4 \%$ of the B-SR and $37.6 \%$ of the U-SR correct responses were given in incorrect positions, the differences favoring $\mathrm{FR}$ over SR performance were more than tripled when the latter included only items given in correct positions. However, neither these percentages nor the absolute greater number of such misplaced responses under B-SR (37.9) than U-SR (27.8) conditions approached significance, and both the overall B-U difference and interaction remained highly significant when these responses were subtracted from the 
SR total-response measures. The B-SR condition also involved over twice as many cases as did the U-SR where two (or more) responses were given in incorrect positions but correct relative order, although this difference was nonsignificant.

Performance on the postexperimental recognition test was evaluated by subtracting the number of incorrect items checked from the number of correct items checked. Except for a nonsignificant $(F<1)$ superiority of FR (11.1) over SR (10.7) conditions, the recognition results followed closely the recall differences, with U-SR (8.7) markedly inferior to B-SR (12.7), B-FR (11.6), and U-FR (10.6) conditions, and highly significant $(p<.001)$ overall $B-U$ difference as well as a significant $(\mathrm{p}<.05) \quad \mathrm{B}-\mathrm{U}$ by $\mathrm{FR}-\mathrm{SR}$ interaction. Discussion

The present results clearly show that either or both sequential blocking of the three letter orders of each set, and/or permitting $S$ free recall of the items in any order, produce marked facilitative effects in recall performance with the present materials. Only under the present U-SR condition, where Ss were forced to recall the items in the ungrouped order in which they were presented, was substantial recall difficulty observed.

However, free recall produced if anything a slight inhibitory effect as compared with serial recall under blocked conditions, in support of Waugh (1961), although her finding of a greater intertrial improvement and increasingly superior performance on later trials for SR than FR conditions was not replicated with the present B conditions. Moreover, the present inferiority of SR to FR performance under U conditions indicates Waugh's results to be limited to conditions where there is no marked discrepancy between order of presentation and preferred order of recall.

Further evidence that recall order was primarily important as a performance rather than a learning variable, and was of lesser importance than blocking in the present task, is provided by (a) the significant superiority of subsequent recognition scores for $G$ over $U$ but not FR over SR conditions, and (b) the sizable G over U superiority remaining under FR conditions. This latter result is consistent with previous demonstrations of a significant blocking effect for categorically related materials in multi-trial freerecall tasks (e.g., Bourne \& Parker, 1964; Dallett, 1964), although its insignificant reversal on the present first trial under $B$ conditions is more comparable with Cofer, Bruce, \& Reicher's (1966) finding of no B-U difference with low-frequency categorized words on a single-trial FR task.

The impressive magnitude of the present $\mathrm{B}-\mathrm{U}$ differences, and also of the B-U by FR-SR interaction, indicates the present mode of varying materials in formal similarity to be a sensitive and potentially useful technique for the investigation of organizational processes in free and serial recall tasks.

\section{References}

Battig, W. F., Merikle, P. M., \& Schild, M. E. Anagram free-recall and recognition learning, and paired-associate transfer. J. verbal Learn. verbal Behav., 1965, 4, 44-52.

Bourne, L. E., Jr., \& Parker, B. K. Interitem relationships, list structure, and verbal learning. Canad. J. Psychol., 1964, 18, 52-61.

Cofer, C. N., Bruce, D. R., \& Reicher, G. M. Clustering in free recall as a function of certain methodological variables. $J$. exp. Psychol., 1966, in press.

Dallett, K. M. Number of categories and category information in free recall. J. exp. Psychol., 1964, 68, 1-12.

Tulving, E. Free recall. In D. L. Horton \& T. R. Dixon (Eds,; Verbal behavior theory and its relation to general $S-R$ theory. Englewood Cliffs, N. J.: Prentice-Hall, in press.

Waugh, N. C. Free vs. serial recall. J. exp. Psychol., 1961, 62, 496-502.

\section{Note}

1. This research was supported by $P$ ublic Health Service Research Grant HD- 01062 from the National Institute of Child Health and Human Development. The author is also indebted to Janis Hagstrom for her extensive contributions to the tabulation and analysis of the data. 